\title{
Graphene-silicene bilayer: A nanocapacitor with permanent dipole and piezoelectricity effect
}

\author{
F. Peymanirad, M. Neek-Amal, ${ }^{*}$ and J. Beheshtian \\ Departement of Physics, Shahid Rajaee Teacher Training University, Lavizan, Tehran 16788, Iran \\ F. M. Peeters \\ Departement Fysica, Universiteit Antwerpen, Groenenborgerlaan 171, B-2020 Antwerpen, Belgium
}

(Received 30 June 2015; published 9 October 2015)

\begin{abstract}
Using density functional theory, we study the electronic properties of a graphene-silicene bilayer (GSB). A single layer of silicene binds to the graphene layer with adhesion energy of about $25 \mathrm{meV} /$ atom. This adhesion energy between the two layers follows accurately the well-known $-1 / z^{2}$ dispersion energy as found between two infinite parallel plates. In small flakes of GSB with hydrogenated edges, negative charge is transferred from the graphene layer to the silicene layer, producing a permanent and a switchable polar bilayer, while in an infinite GSB, the negative charge is transferred from the silicene layer to the graphene layer. The graphene-silicene bilayer is a good candidate for a nanocapacitor with piezoelectric capabilities. We found that the permanent dipole of the bilayer can be tuned by an external perpendicular electric field.
\end{abstract}

DOI: 10.1103/PhysRevB.92.155113

PACS number(s): 73.22.Pr

\section{INTRODUCTION}

Graphene-like materials have been gaining increasing popularity over the past few years. Heterostructures and devices can be made by stacking different two-dimensional (2D) crystals on top of each other [1-5]. The 2D allotrope of graphite and the quasi-2D allotrope of silicon, i.e., graphene and silicene, have many interesting and extraordinary properties and can be stacked on top of each other to form a new type of heterostructure, i.e., a graphene-silicene heterostructure. Their properties are affected by the number of layers, the external shear stress, and the stacking structure. Monolayer graphene is a gapless semimetal, while silicene has a tiny energy gap. However, there are indications that the realization of freestanding silicene is doubtful [6-8]. However, it is possible to intercalate a Si layer at the interface between epitaxially grown graphene on metal crystal surfaces, e.g., Ru and Ir, while the graphene crystallinity is maintained [9]. An energy gap in both graphene and silicene can be induced by using different methods: by cutting the flake, (i.e., quantum confinement effect) or doping by other elements such as nitrogen and boron [10,11]. Moreover, the electronic gap of a graphene bilayer (or other 2D materials) can be controlled externally by applying a perpendicular electric field which was clearly seen in the quantum Hall regime [12,13]. Recently Nigam et al. [14] used density functional theory to study heterostructures consisting of silicene, graphene, and BN monolayers and investigated the effect of a perpendicular electric field. They predicted an asymmetric charge density around the graphene-silicene bilayer which motivated us to investigate the polarization properties and possible ferroelectricity in a large flake of graphene-silicene bilayer. Moreover, Sante et al. [15] recently found that, in honeycomb binary compounds, dipoles arise from the buckled structure, where the A and B ions are located on the sites of a bipartite corrugated honeycomb lattice with trigonal symmetry, e.g., for the SiGe binary compounds a dipole of $0.88 \times 10^{-12} \mathrm{C} / \mathrm{m}$ per unit area was found [15].

*neekamal@srttu.edu
In this study, using density functional calculations, we report on the electronic and structural properties of a flake and an infinite graphene-silicene bilayer (GSB) and obtain the adhesion energy between the two layers. We find that GSB has a permanent dipole which makes it a good candidate for a polar bilayer as well as for a ferroelectric nanocapacitor. The polarization can be tuned by a perpendicular external electric field. The molecular polarizability of the GSB is found to be anomalously negative which makes the system equivalent to a plasmonic media. We also present a model for the piezoelectricity effect in an infinite GSB which is also relevant for flakes.

This paper is organized as follows: In Sec. II, we introduce the model and outline the computational method. Results and discussions are presented in Sec. III which contains the following six sections: the lattice structure of the graphenesilicene bilayer, charge distribution and intrinsic polarization, the energy gap between the highest occupied molecular orbital and the lowest unoccupied molecular orbital (HOMO-LUMO energy gap), adhesion energy, polarizability effects of GSB, and the piezoelectricity effect in infinite GSB. The paper is summarized in Sec. IV.

\section{MODELS AND COMPUTATIONAL METHODS}

Our system is comprised of a finite-size hexagonal flake of graphene and a finite-size hexagonal flake of silicene (114 $\mathrm{C}$ and $54 \mathrm{Si}$ atoms), which were passivated by hydrogen atoms at the edges [Fig. 1(a)]. We also performed additional calculations for a smaller (and larger) flake that contains $54 \mathrm{C}$ (222 C) and $24 \mathrm{Si}(114 \mathrm{Si})$ atoms and for an infinite system. Notice that, because of the lattice mismatch between graphene and silicene and the non-flat structure of silicene, generating a perfect unit cell and performing traditional periodic-boundary calculations is infeasible without inducing internal strain. In Ref. [14], a unit cell (having $18 \mathrm{C}$ and $8 \mathrm{Si}$ atoms) was used with co-periodic boundary conditions. Because of the mismatch between the graphene and silicene lattices, such an approach introduces internal strain in the heterostructure, modifying, e.g., the band structure. We show that our results for a flake 


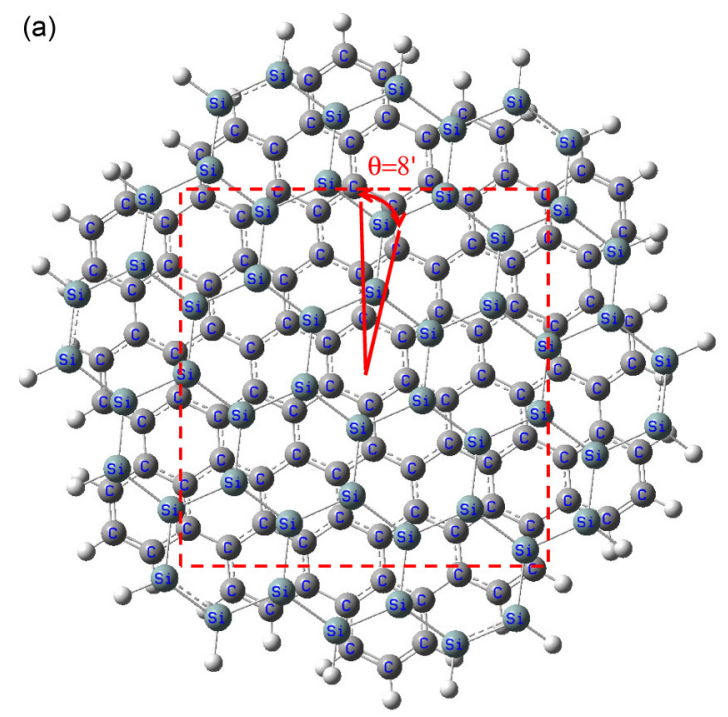

(c)

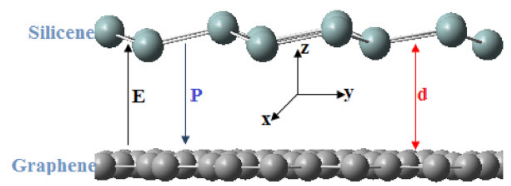

(b)
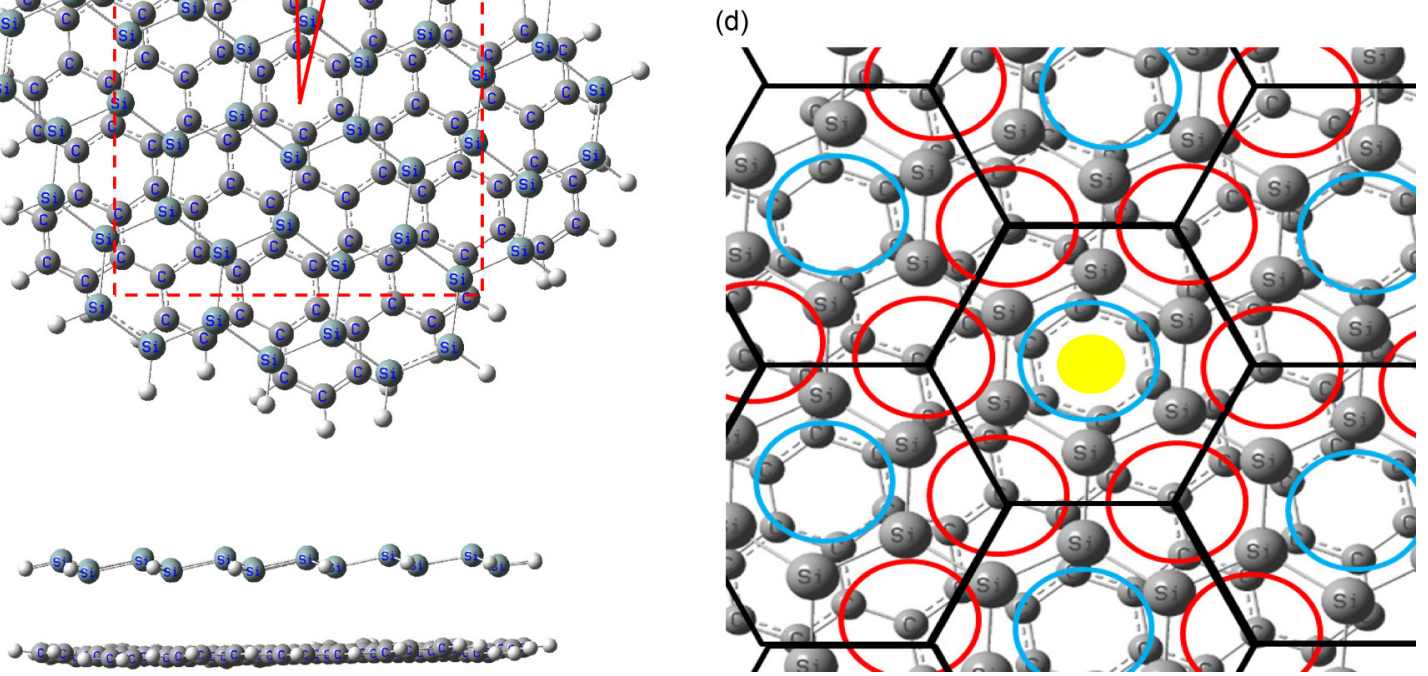

FIG. 1. (Color online) (a), (b) Top and side view of the optimized graphene-silicene bilayer (the white atoms at the edges refer to the $\mathrm{H}$ atoms). (c), (d) Side and top view of the portion inside the red rectangle of panel (a). The thick solid black lines in panel (d) indicate the moiré pattern and the red or blue circles are guides to the eye in order to see more clearly the stacked structure. The large (small) dots refer to $\mathrm{Si}(\mathrm{C})$ atoms.

of graphene-silicene bilayer are very different. We optimize our system by using the M06-2X functional and the 6-31G* basis set. The M06-2X functional is selected because it is able to quantify the dipole moment and estimate the van der Waals interaction between the layers [16]. We also performed periodic-boundary-condition calculations for an infinite GSB for comparison purposes.

\section{RESULTS AND DISCUSSIONS}

\section{A. Graphene-silicene bilayer structure}

First, we optimize separately the graphene and silicene flakes and subsequently the graphene-silicene bilayer is optimized. In our calculations, the interlayer binding energy is defined as the atom-averaged energy difference between the separated layers and the bound layers as

$$
E_{\mathrm{vdW}}=\frac{E_{\mathrm{GSB}}-E_{\mathrm{Si}}-E_{\mathrm{G}}}{N},
$$

where $\mathrm{E}_{\mathrm{vdW}}$ represents the interlayer binding energy in units of $\mathrm{meV}$ /atom, $\mathrm{E}_{\mathrm{bilyer}}$ is the total energy of GSB in the M06-2X model, $E_{\mathrm{Si}}$ and $E_{\mathrm{G}}$ corresponds to the energy of the isolated single layer of silicene and graphene, respectively and $N$ is the number of atoms in the GSB.

The vdW interaction between the two layers keeps the silicene flake stable into a buckled structure [see Figs. 1(a)-1(d)], i.e., the final optimized GSB is a flat graphene flake and a buckled silicene flake (with $0.43 \AA$ buckling height) which are separated by $3.686 \AA$. This distance allows electron transfer from one layer to the other [17]. In Fig. 1(d) we show the corresponding moiré pattern formed in the GSB. The blue and red circles refer to different arrangements of the $\mathrm{C}$ atoms inside the silicene hexagons. The yellow spot refers to the center of the hexagon flake in order to guide the eye. The central hexagon of carbon atoms is rotated by about $\theta=8^{\circ}$ with respect to the silicene hexagon, called the moiré angle. Our finding for the minimum-energy configuration, shown in Fig. 1(d), is different from the one proposed in Ref. [14], where they chose an incomplete unit cell (which induced an external boundary stress because of the $3 \%$ lattice mismatch) and the misorientation angle was missed. In order to compare the energy of the rotated graphene over silicene with respect to the aligned one we performed an additional single-point energy calculation for $\theta=0^{\circ}$. We found that the total energy for the rotated system is $6.320 \mathrm{eV}$ (corresponding to $4 \%$ lower energy) lower than that of the aligned configuration (for our small flake). We also performed additional DFT calculations, with periodic boundary conditions, using the unit cell that is taken from Fig. 1(a) and found that the rotated structure is more stable than the nonrotated one (four unit cells of the PBC calculations are shown in Fig. 2). The lattice mismatch in our unit cell is less than $1.4 \%$ which is half of that in Ref. [14]. Next, we focus on the results of the finite-size (i.e., flake) system.

\section{B. Charge distribution over graphene-silicene bilayer}

The different electronegativity of $\mathrm{C} / \mathrm{Si}$ and $\mathrm{H}$ atoms causes a charge redistribution at the edge of the graphene and silicene flakes which are both terminated by $\mathrm{H}$ atoms, e.g., $\mathrm{C}$ atoms 
(a)

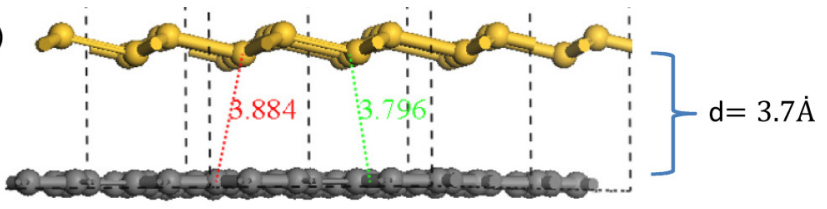

(b)

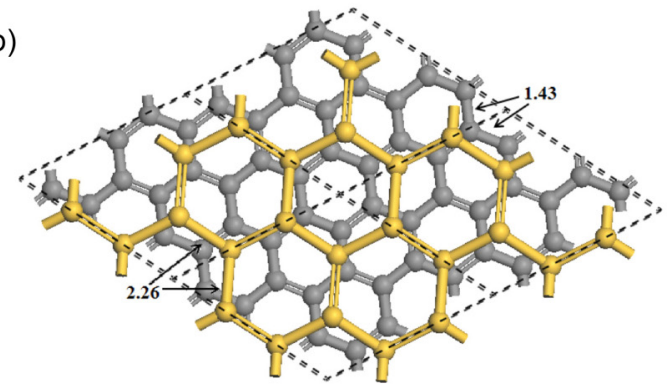

(c)

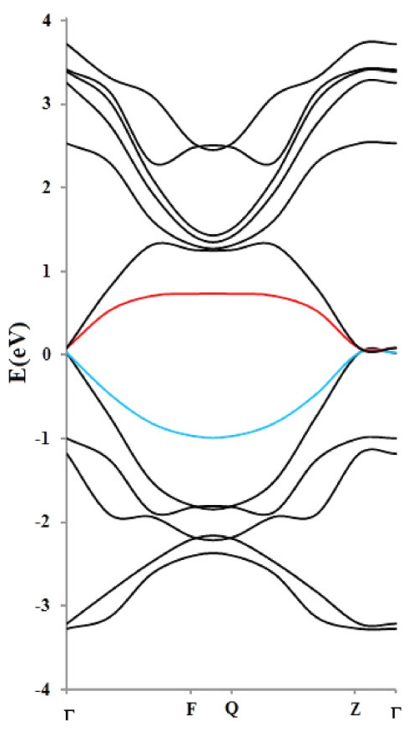

FIG. 2. (Color online) (a) Side and the top view of the unit cell used for the periodic boundary calculations. (b) The corresponding band structure. The valance and conductance bands are shown by blue and red curves, respectively.

deplete the hydrogen atoms and adsorb their charge. Thus, all hydrogen atoms have almost equal positive charges. The graphene and silicene layer are neutral before they are put on top of each other. The charges over each layer are listed in Table I. However in the bilayer system, a large amount of charge $(Q)$ is transferred from the graphene flake to the silicene flake resulting in a polarized bilayer (a giant polar molecule consisting of more than 168 atoms). The total charge transferred from the graphene to the silicene flake is found to be $Q=0.93 e(0.5 e)$ for the large (small) flake. Thus, the silicene (graphene) layer becomes negatively (positively) charged. It is very important to note that (as seen from the numbers written in brackets in Table I) in the individual (between the) flakes the charge distribution (charge transfer) is strongly (weakly) affected by the edge atoms, i.e., the hydrogens. Note that, after optimization, the absolute value of the total charge over each layer is equal. It is also worth to mentioning that, in our PBC calculations, we found positive (negative) charge over the silicene (graphene) layer of about $0.021 e$ per unit

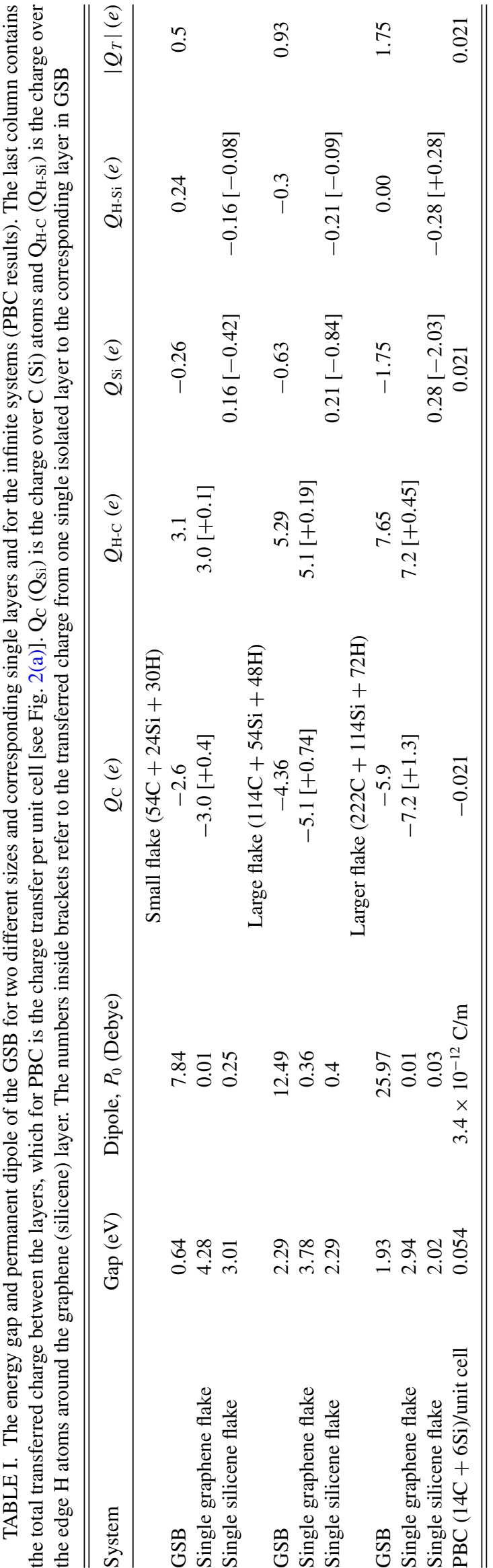


cell which has a different sign and value than that of our finite-size systems. The latter shows the important role of the edge atoms in the electronic structure of flakes and ribbons. The PBC calculations indicate that an infinite GSB also has a permanent dipole, i.e., 0.4 Debye per unit cell. This charge over the layers causes an electrostatic potential difference between the two layers and consequently the opening of an energy band gap. The energy band gap from our band structure calculations [see Fig. 2(b)] is found to be $54 \mathrm{meV}$ which is close to $51 \mathrm{meV}$ reported in Ref. [14]. The band structure and reciprocal lattice points are very different than that for a hexagonal lattice structure. The buckled height found from $\mathrm{PBC}$ calculations is $0.57 \AA$ and the $\mathrm{C}-\mathrm{C}(\mathrm{Si}-\mathrm{Si})$ bond length is $1.43 \AA(2.26 \AA)$. Obviously, because of the breaking of the inversion symmetry in the infinite GSB, it is a piezoelectric material. Unlike bilayer graphene with a $4 \times 4$ Hamiltonian, the tight-binding Hamiltonian for GSB is $20 \times 20$ dimensional which makes tight-binding calculations almost impractical.

\section{HOMO-LUMO}

Here we present the electronic ground-state properties of GSB. The highest occupied molecular orbitals (HOMOs) and the lowest unoccupied molecular orbitals (LUMOs) give information about the chemical activity of the system and possible charge transfer. As can be seen from Fig. 3, the HOMO (LUMO) is only distributed over graphene (silicene); see Figs. 3(a) and 3(b). This effect is independent of the chosen exchange-correlation functional (we found the same distribution using B3LYP). The energy gap $\left(E_{\text {HOMO }}-E_{\text {LUMO }}\right)$ and the corresponding dipole moment of the system are listed in Table I. The Fermi energy of the system can be found by using $E_{\mathrm{F}}=\left(E_{\mathrm{HOMO}}+E_{\mathrm{LUMO}}\right) / 2$. The Fermi energy $E_{\mathrm{F}}$ of a single layer of graphene $(-3.78 \mathrm{eV})$ is larger than that of silicene $(-4.65 \mathrm{eV})$, therefore we would expect charge transfer from graphene to silicene. It is interesting to note that the latter causes a giant permanent dipole moment of about $P_{0} \simeq 12.5$ Debye $\left(14.4 \times 10^{-12} \mathrm{C} / \mathrm{m}\right)$ perpendicular to the layers [see the blue arrow in Fig. 3(c)]. The obtained dipole for the larger flake $\left(14.0 \times 10^{-12} \mathrm{C} / \mathrm{m}\right)$ is one order of magnitude larger than that found in Ref. [15] $\left(0.88 \times 10^{-12} \mathrm{C} / \mathrm{m}\right)$ for $\mathrm{SiGe}$

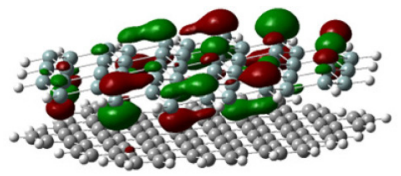

(a)

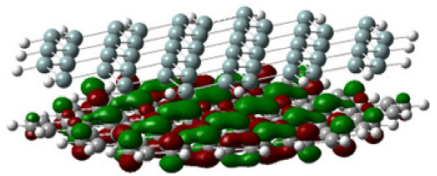

(b) (c)

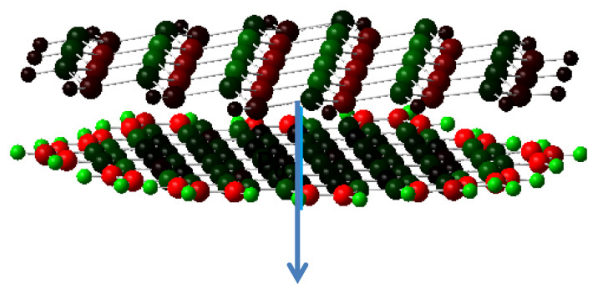

FIG. 3. (Color online) Frontier molecular orbitals in GSB. (a) HOMO, (b) LUMO, and (c) dipole moment in the optimized flake of GSB. honeycomb binary compounds and is of the order of InAr dipole $\left(11.1 \times 10^{-12} \mathrm{C} / \mathrm{m}\right)$ which is an indication of a strong polarization effect in the GSB (note that the obtained dipole from $\mathrm{PBC}$ calculations is $3.4 \times 10^{-12} \mathrm{C} / \mathrm{m}$ ). The larger atomic radius and the static polarizability of the $\mathrm{Si}$ atoms with respect to the $\mathrm{C}$ atoms and the buckling structure of silicene enhance the polarization effects. In fact, the charge $Q$ is transferred from the graphene sheet to silicene which causes the system to be polarized. Neglecting the buckling effect in the silicene flake, one can approximately write $P_{0} \cong Q d$ where $d$ is the equilibrium interlayer distance between the layers. We expect that, by increasing the size of the flake of GSB, $P_{0}$ will increase (we performed additional DFT calculations for a smaller flake and found $P_{0} \simeq 7.84$ Debye, see Table I). Thus the relevant quantity for an infinite bilayer would be $D=P_{0} / V$ where $V$ is the volume between the two layers of GSB.

Moreover, the energy gap of the larger (smaller) flake is found to be $2.296 \mathrm{eV}(0.637 \mathrm{eV})$. In Table I we list the energy gap, the dipole moment, and the total transferred charge from graphene to silicene and corresponding values for the isolated silicene and graphene flakes.

\section{Binding energy}

The binding energy stored between the two layers (i.e., the adhesion energy) is an important quantity. We start from two optimized layers then move one layer with respect to the other and perform energy calculations. The results are shown in Fig. 4. The minimum energy is found to be about $-25 \mathrm{meV} /$ atom $(-22 \mathrm{meV} /$ atom $)$ for the unit cell of the PBC calculations (large flake) with interlayer distance $3.8 \AA$ (3.7 $\AA$ ). The minimum energy is comparable to the adhesion energy of the graphene-boron nitride bilayer, i.e., 30 to $40 \mathrm{meV} /$ atom [18]. The dispersion energy between two infinite plates (see the inset of Fig. 4) is given by

$$
U \cong \frac{A}{z^{9}}-\frac{B}{z^{2}},
$$

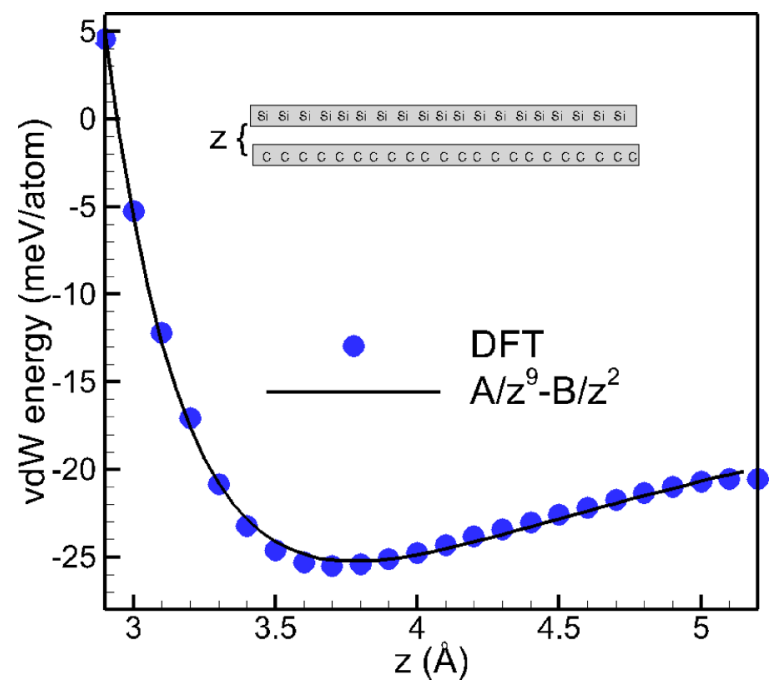

FIG. 4. (Color online) The variation of the binding energy of the graphene-silicene bilayer with interlayer distance. Solid line is the best fit to $A / z^{9}-B / z^{2}$. 
where $B$ is the Hamaker constant [19]. Equation (1) fits reasonably well our DFT results (see the curve in Fig. 4). The Hamaker constant for the studied PBC unit cell (large flake) is found to be $B=290.5 \mathrm{meV} \AA^{6} /$ atom $\left(1096 \mathrm{meV}^{6} /\right.$ atom). Notice the excellent fit having infinitesimal $(\sim 0.001)$ standard error.

\section{E. Polarizability and capacitance}

The presence of a permanent dipole in the GSB system implies that we have an atomic thin polar nanoscale flake. This is a nanoscale capacitor which stores energy of about $\sim 25 \mathrm{meV} /$ atom and an electrostatic energy per volume $\frac{1}{2} \frac{D^{2}}{\epsilon_{0}}$ (if we treat the system classically the capacitance is $\frac{\epsilon_{0} A_{0}}{d}$ ). As already mentioned the obtained interlayer distance $(d=3.7 \AA)$ and the dipole moment from DFT calculations help us to approximate the total charge $Q$ over each plate using $P_{0} \simeq$ $Q d$, e.g., for the larger flake $Q= \pm \frac{12.5 \text { Debye }}{0.37 \mathrm{~nm}} \simeq \pm 0.7 e$ which is $25 \%$ less than $0.93 e$ found from DFT calculations (the difference might be due to the neglect of the buckling effect in the latter equation and the edge effects). The corresponding electrostatic potential difference and the electric field between the two flakes are given by

$$
\Delta V_{0}=\frac{\langle P\rangle}{\epsilon_{0}}, \quad E_{0}=d \frac{\langle P\rangle}{\epsilon_{0}},
$$

where $\langle P\rangle=P_{0} / A$ is the polarization per unit area. For the studied large (small) flake we find $\Delta V_{0} \simeq 1.7$ (2.0) $\mathrm{V}$. The polarization of the system can be changed by an external electric field. By applying a perpendicular electric field (a schematic model is shown in Fig. 5) we found the polarizability of the GSB by using the following equation:

$$
P=P_{0}+\alpha E,
$$

where $\alpha$ is the polarizability of the system, $E$ is the external electric field, and $P_{0}$ is the initial dipole of the system. Figure 6 shows the variation of the net dipole moment (for the smaller flake) as function of the applied electric field. The polarizability of the system is found to be negative, i.e., $\alpha=-0.367$ Debye $\AA / \mathrm{V}$. The electric field changes the charge distribution over the system as well as its polarization. The external electric field along the $z$ direction $(-z)$ causes the opposite polarization (parallel) in the system which results in a decrease (increase) of the total $P$. The negative polarizability reminds us of the polarizability of a two-dimensional electron gas in the presence of an external electric field. The interlayer distance and the charge over each layer change linearly with slopes $-0.37 \AA^{2} / \mathrm{V}$ [see Fig. 6(c)] and $-0.64 \mathrm{e} \AA / \mathrm{V}$ [see Fig. 6(b)], respectively. Therefore, by increasing the electric field, more charge is transferred from graphene to silicene resulting in a larger dipole moment.

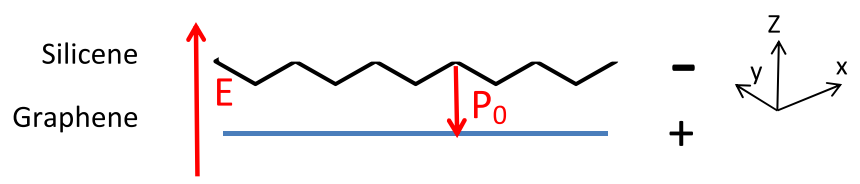

FIG. 5. (Color online) The schematic model for a flake of GSB in the presence of an upward external electric field.

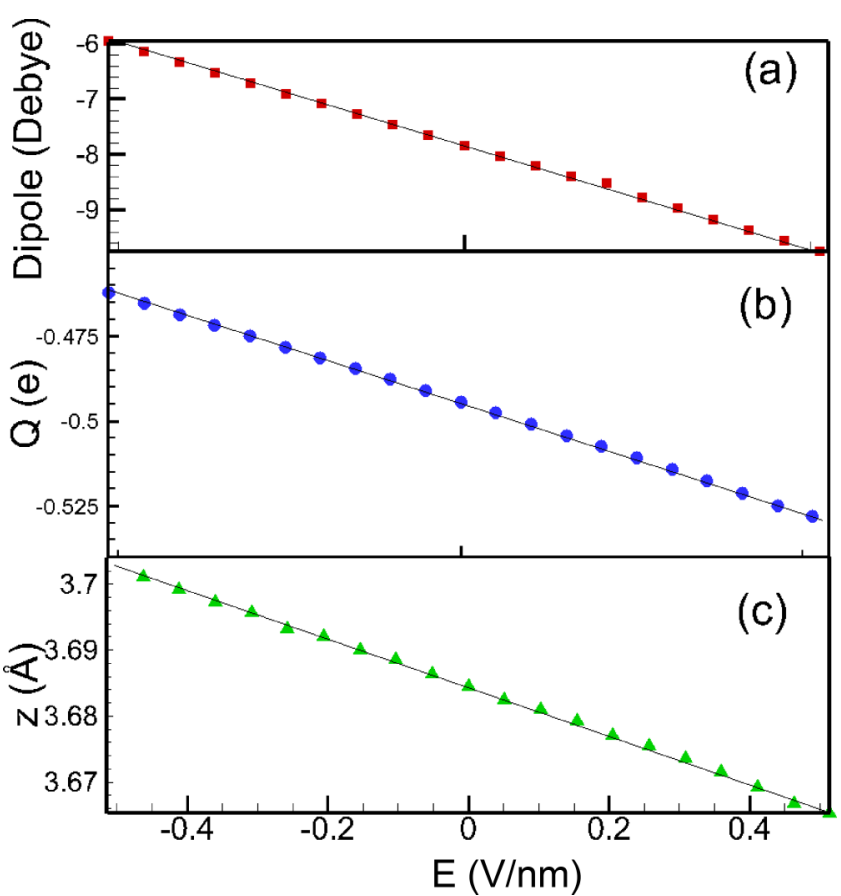

FIG. 6. (Color online) The variation of (a) electric dipole moment, (b) transferred charge, and (c) interlayer distance with applied electric field perpendicular to the GSB layers (small flake).

Thus, the GSB exhibits a piezoelectric effect; namely, by changing the interlayer distance, which is equivalent to applying an external perpendicular pressure, we found a change in the dipole moment and concomitant transfer of charge. The results are shown in Fig. 7. It is seen that the absolute value of the dipole moment increases nonlinearly against the interlayer distance [Fig. 7(a)], which is an indication of the sensitivity of the GSB to external pressure. We were able to fit the cubic function $P=0.95 z^{3}-15.95 z^{2}+89.00 z-167.74$ [solid curve in Fig. 7(a)]. As can be seen from Fig. 7(b) the dipole moment changes nonlinearly with the force (force $=$ $-d U / d z$ ) (we used a numerical differentiation of our DFT data shown in Fig. 4) which is an indication of a nonlinear piezoelectricity effect in the GSB.

\section{PIEZOELECTRICITY EFFECT IN INFINITE GRAPHENE-SILICENE BILAYER}

Finally, we turn our attention to the calculation of the piezoelectricity effect for an infinite GSB. Assuming a linear variation of the dipole (perpendicular to the plates) with interlayer distance $z$, i.e., $P_{z}=P_{0}+\beta(z-d)$, where $d=3.8 \AA$ is the equilibrium distance, and the adhesion energy of the form $U(z)=A / z^{m}-B / z^{n}$, the force between the plates is given by $F(z)=m A / z^{m+1}-n B / z^{n+1}$. The $z$ component of the stress tensor per unit cell is written as $\sigma_{z z}=\left(m A / z^{m+1}-n B / z^{n+1}\right) / S$, where $S$ is the area of the unit cell. Therefore the nonzero element of the third-rank piezoelectricity tensor (using $P_{z}=e_{3} \sigma_{z z}$ ) is 

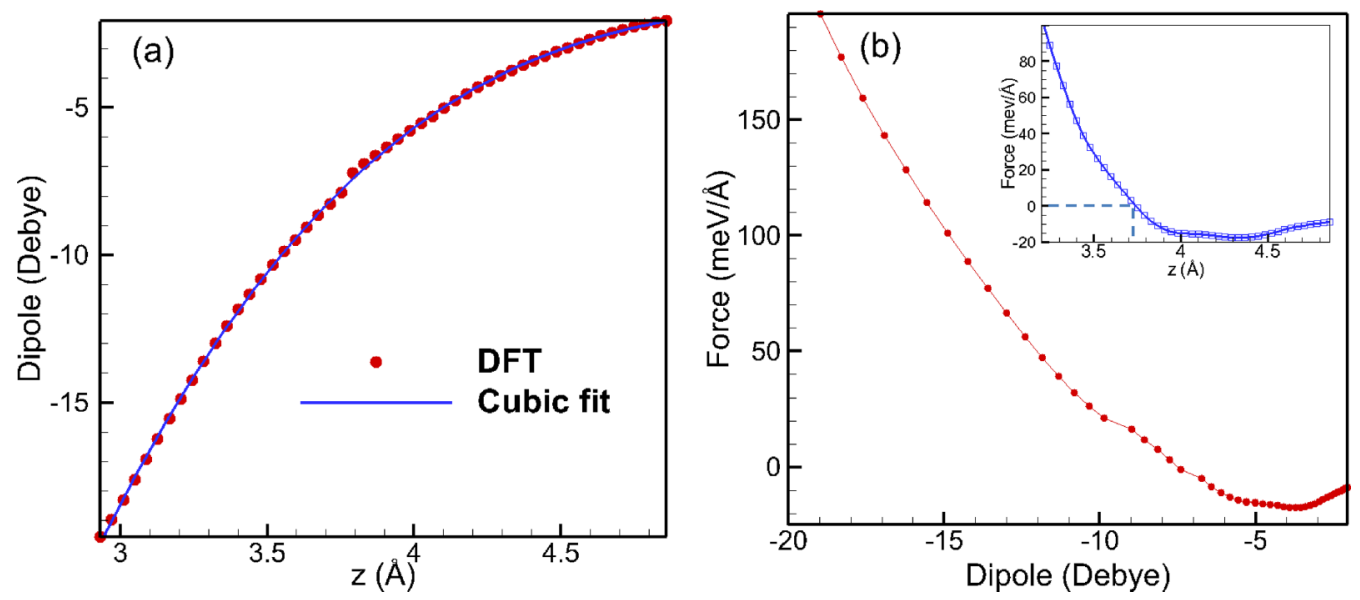

FIG. 7. (Color online) (a) The variation of the induced dipole with the interlayer distance between the graphene and silicene layers. (b) The nonlinear variation of the force versus the dipole moment. The inset shows the variation between force and distance.

found to be

$$
e_{3}(z)=\frac{S z^{m+n+1}\left(P_{0}+\beta(z-d)\right)}{m A z^{n}-B n z^{m}},
$$

which is a nonlinear function of the interlayer distance. For $m=9$ and $n=2$ it is simplified to

$$
e_{3}(z)=\frac{S z^{12}\left(P_{0}+\beta(z-d)\right)}{9 A z^{2}-2 B z^{9}} \text {. }
$$

\section{CONCLUSIONS}

In summary we investigated the electronic properties of a finite-size graphene-silicene bilayer by using density functional theory. We found that the stacked graphene-silicene bilayer has a size-dependent intrinsic polarization with a dipole moment of about $3.4 \times 10^{-12} \mathrm{C} / \mathrm{m}$ which can be tuned by applying an external perpendicular electric field. We found that the graphene-silicene bilayer is a good candidate for a ferroelectric nanocapacitor with permanent dipole. An infinite GSB shows ferroelectric properties but with opposite sign for the dipole as compared to the finite flake. This is a consequence of the hydrogen termination of the graphene and silicene flakes. The dipole moment is a function of the GSB flakes which changes sign as function of the size of the GSB flake. The available DFT software does not allow us to determine the GSB size at which the dipole moment changes sign. The dispersion energy between the graphene and silicene flake follows the well-known $-1 / z^{2}$ relation. We showed that the GSB is piezoelectric and for the infinite GSB we obtained an analytic expression for the piezoelectric third-rank tensor element.

\section{ACKNOWLEDGMENT}

This work was supported by the ESF-Eurographene project CONGRAN and the Flemish Science Foundation (FWO-V1).
[1] K. S. Novoselov, A. K. Geim, S. V. Morozov, D. Jiang, Y. Zhang, S. V. Dubonos, I. V. Grigorieva, and A. A. Firsov, Science 306, 666 (2004).

[2] A. K. Geim and K. S. Novoselov, Nat. Mater. 6, 186 (2007).

[3] A. K. Geim, Science 324, 1530 (2009).

[4] A. K. Geim and I. V. Grigorieva, Nature (London) 499, 419 (2013).

[5] H. Fang, C. Battagliaa, C. Carraro, S. Nemsak, B. Ozdol, J. S. Kang, H. A. Bechtel, S. B. Desai, F. Kronast, A. A. Unal, G. Conti, C. Conlon, G. K. Palsson, M. C. Martin, A. M. Minor, C. S. Fadley, E. Yablonovitch, R. Maboudian, and A. Javey, Proc. Natl. Acad. Sci. U. S. A. 111, 6198 (2014).

[6] Y. Feng, D. Liu, B. Feng, X. Liu, L. Zhao, Z. Xie, Y. Liu, A. Liang, C. Hu, Y. Hu, S. He, G. Liu, J. Zhang, C. Chen, Z. Xu, L. Chen, K. Wu, Y.-T. Liu, H. Lin, Z.-Q. Huang, C.-H. Hsu, F.-C. Chuang, A. Bansil, and X. J. Zhou, arXiv:1503.06278.

[7] M. Neek-Amal, A. Sadeghi, G. R. Berdiyorov, and F. M. Peeters, Appl. Phys. Lett. 103, 261904 (2013).
[8] Kh. Shakouri, P. Vasilopoulos, V. Vargiamidis, and F. M. Peeters, Phys. Rev. B 90, 235423 (2014).

[9] J. Mao, L. Huang, Y. Pan, M. Gao, J. He, H. Zhou, H. Guo, Y. Tian, Q. Zou, L. Zhang, H. Zhang, Y. Wang, S. Du, X. Zhou, A. H. C. Neto, and H.-J. Gao, Appl. Phys. Lett. 100, 093101 (2012); L. Meng, R. Wu, H. Zhou, G. Li, Y. Zhang, L. Li, Y. Wang, and H.-J. Gao, ibid. 100, 083101 (2012).

[10] J. Beheshtian, A. Sadeghi, M. Neek-Amal, K. H. Michel, and F. M. Peeters, Phys. Rev. B 86, 195433 (2012).

[11] Y.-C. Chen, D. G. de Oteyza, Z. Pedramrazi, C. Chen, F. R. Fischer, and M. F. Crommie, ACS Nano 7, 6123 (2012).

[12] E. V. Castro, K. S. Novoselov, S. V. Morozov, N. M. R. Peres, J. M. B. Lopes dos Santos, J. Nilsson, F. Guinea, A. K. Geim, and A. H. Castro Neto, Phys. Rev. Lett. 99, 216802 (2007).

[13] Q. Liu, L. Li, Y. Li, Z. Gao, Z. Chen, and J. Lu, J. Phys. Chem. C 116, 21556 (2012).

[14] S. Nigam, S. K. Gupta, C. Majumder, and R. Pandey, Phys. Chem. Chem. Phys. 17, 11324 (2015). 
[15] D. Di Sante, A. Stroppa, P. Barone, M.-H. Whangbo, and S. Picozzi, Phys. Rev. B 91, 161401(R) (2015).

[16] S. M. Seyed-Talebi and M. Neek-Amal, J. Appl. Phys. 116, 153507 (2014).

[17] O. Leenaerts, B. Partoens, and F. M. Peeters, Phys. Rev. B 77, 125416 (2008).
[18] B. Sachs, T. O. Wehling, M. I. Katsnelson, and A. I. Lichtenstein, Phys. Rev. B 84, 195414 (2011).

[19] S. A Safran, Statistical Thermodynamics of Surfaces, Interfaces and Membranes, Frontiers in Physics, edited by D. Pines (Addison-Wesley, Reading, 1994). 